\title{
Opinión de universitarios bolivianos sobre el uso de alcohol en el contexto universitario
}

\author{
Nelly Balda-Cabello ${ }^{1}$ \\ Edilaine Cristina da Silva²
}

\begin{abstract}
Se trata de una investigación cualitativa que enfocó el mundo universitario sobre los valores de vida utilizados por los jóvenes para lidiar con los peligros del consumo de alcohol. Fueron realizados grupos de discusión con 46 jóvenes universitarios. Los resultados evidenciaron que la sociedad boliviana es tolerante y permisiva en el consumo de alcohol de universitarios hombres. Los valores y el amor propio, el respeto por sí mismo, la responsabilidad y la capacidad de reflexión constituyeron un marco de actuación regulador, sin embargo no es generalizado.
\end{abstract}

Descriptores: Valores Sociales; Estilo de Vida; Consumo de Bebidas Alcohólicas; Estudiantes.

\footnotetext{
${ }^{1}$ Pedagoga, Investigadora. Profesor Titular, Universidad Mayor de San Andrés, La Paz, Bolívia. E-mail: nellybalda@yahoo.es. 2 Enfermera, Doctora en Enfermería. Profesor Doctor, Escola de Enfermagem de Ribeirão Preto, Universidade de São Paulo, Centro Colaborador de la OMS para el Desarrollo de la Investigación en Enfermería, SP, Brasil. E-mail: nane@eerp.usp.br.
}

Correspondencia:

Edilaine Cristina da Silva

Universidade de São Paulo. Escola de Enfermagem de Ribeirão Preto

Departamento de Enfermagem Psiquiátrica e Ciências Humanas

Av. dos Bandeirantes, 3900

Bairro: Monte Alegre

CEP: 14040-902 Ribeirão Preto, SP, Brasil

E-mail: nane@eerp.usp.br 


\section{Opinião de universitários bolivianos sobre o uso de álcool no contexto universitário}

Esta é uma investigação qualitativa que enfocou o mundo universitário sobre os valores de vida, utilizados pelos jovens, para lidar com os perigos do consumo de álcool. Foram realizados grupos de discussão com 46 jovens universitários. Os resultados evidenciaram que a sociedade boliviana é tolerante e permissiva para o consumo de álcool de universitários homens. Os valores e o amor próprio, o respeito por si mesmo, a responsabilidade e a capacidade de reflexão constituíram marco de atuação regulador, porém, não é generalizado.

Descritores: Valores Sociais; Estilo de Vida; Consumo de Bebidas Alcoólicas; Estudantes.

\section{Bolivian University Students' View of Alcohol Use in the University Context}

Qualitative research inquiring into the university world about the values of young people's life to successfully offset the danger of alcohol consumption. Discussion groups were implemented in workshops with 46 university students. The results show that that Bolivian society is very tolerant and permissive towards alcohol consumption by male university students. Values like pride, self-respect, responsibility and capacity for reflection constitute a framework of regulatory action, but it is not generalized.

Descriptors: Social Values; Life Style; Alcohol Drinking; Students.

\section{Introducción}

Hoy, la salud es percibida por todos los segmentos poblacionales como un elemento fundamental del capital humano, indispensable para el logro del desarrollo humano sostenible. Pese a ello, en países como Bolivia aún se requiere insistir enfáticamente en políticas públicas y servicios de salud para personas jóvenes, que atiendan problemáticas específicas como la prevención del consumo de alcohol, que se sustente en valores y hábitos de vida saludable.

La temática de hábitos de vida saludable en relación al consumo de alcohol en jóvenes universitarios en Bolivia, pese a ser una vertiente interesante ha sido poco explorada. Sin embargo, en los últimos años se han consolidado esfuerzos investigativos generados por el Centro Latino Americano de Investigación Científica (CELIN) con el auspicio del Consejo Nacional de Lucha contra el Tráfico Ilícito de Drogas, la Organización Panamericana de la Salud (OPS) y entidades gubernamentales $^{(1)}$.

El consumo de alcohol por jóvenes universitarios ha aumentado considerablemente en la región y en el país.
En muchos casos, constituye la primera aproximación de los jóvenes al mundo de las drogas. Investigadores del tema, estiman que los consumidores de alcohol tienen alta probabilidad de consumir cocaína alguna vez. De igual manera, establecen la vinculación entre el sujeto bebedor y el círculo cercano. El intervalo de edad en las personas consumidoras de alcohol se sitúa entre los 25 y 35 años y los problemas derivados de su uso afectan por igual a hombres y mujeres. Se requiere que el sistema universitario boliviano cuente con la atención necesaria en el tema de prevención del consumo de drogas. Algunas investigaciones bolivianas recientes, ratifican esta aseveración ${ }^{(1-2)}$.

Las investigaciones referidas a la prevención, han enfatizado en el uso de metodologías cuantitativas. Actualmente se requiere investigaciones de orden cualitativo que evidencien si los valores concebidos como criterios o pautas de conducta, poseen la suficiente fuerza para modelar los actos de los jóvenes. En el sentido, de mostrar las vías de comportamiento que les permita decidir si ingieren alcohol o no, como ejercicio 
de una conciencia que sabe lo que determina para sí y porqué. También, es preciso conocer los hábitos de vida saludable y el papel que éstos juegan como mecanismos de regulación de las vidas jóvenes.

En la investigación los conceptos centrales son valores y hábitos de vida saludables. Para efectos comprensivos, deben ser considerados como conjunto articulado y sinérgico.

Los valores pueden ser entendidos como criterios y pautas de conducta que nos permiten actuar bajo la influencia de una conciencia esclarecida, de una conciencia moral. Los valores aspiran a tener una aplicación universal y compartida por todos los seres humanos. Están ligados a la propia existencia, afectan la conducta, configuran y modelan las ideas, sentimientos y nuestros actos. Son dinámicos, estando ligados al desarrollo de la personalidad y muy influenciados por los procesos de socialización. Los valores no se enseñan, hay que vivirlos.

Los hábitos de vida saludable son el principal parámetro para medir el bienestar y la calidad de vida de un grupo humano en términos de salud. Incluyen entre otros, la instauración y el aumento o disminución de comportamientos relacionados con la condición y actividad física, los hábitos alimenticios, la recreación y el uso adecuado del tiempo libre en actividades que les planteen desafíos para generar confianza en sí mismos y en sus potencialidades. Implica el autocuidado y el cuidado médico y el sueño, así como normas culturales que desalientan el consumo indebido de alcohol.

Comprender la gama de valores, creencias, y hábitos de vida saludable sobre los que gira la vida de los jóvenes universitarios de la ciudad de El Alto, Bolivia, permitirá una lectura explicativa de cómo los valores se constituyen en elementos ordenadores de la actuación social de un joven.

Es imprescindible que la universidad se replantee su papel como formadora de valores en las nuevas generaciones. Con este objetivo de actuación, instaurar en los universitarios la conciencia crítica y la práctica responsable de la autonomía, es un requisito indispensable para una vida ética.

La investigación que se presenta es una investigación cualitativa; indaga en el mundo universitario sobre las fortalezas morales que representan los valores y los hábitos de vida saludable en la vida de los jóvenes, para contrarrestar con éxito los peligros del consumo de alcohol. El análisis de contenido se nutre de los aspectos relevantes que surgen del ejercicio intuitivo o consciente de los valores de los universitarios, de sus miradas sobre sí mismos, de los valores que los guían y de sus maneras de llevar adelante una vida saludable.

\section{Objetivo}

Conocer la opinión de los universitarios sobre el consumo de alcohol e indagar sobre la influencia de los valores y hábitos de su vida cotidiana, en el comportamiento en el uso de drogas.

\section{Metodología}

\section{Tipo de Investigación}

Toda investigación es un comprender e interpretar al otro. En las investigaciones cualitativas, es imprescindible la virtud de la "buena escucha", del aprender a escuchar al otro, de oír y entender para una mejor comprensión de los sujetos, sus discursos y formas de actuación. Entender es ir con el otro, con lo que dice aunque no haya asentimiento, generar diálogos fructíferos que hablen de avenencia. Es un saber oír, buscando que nada pase inadvertido. Es hacer del diálogo una forma de comunicación con sentido y relevancia(3). El análisis se orienta a la interpretación, más que a establecer normas generalizables al conjunto de la población universitaria joven. El presente estudio corresponde a una investigación cualitativa cuya finalidad es observar, describir los fenómenos en una situación concreta de la vida universitaria. Vale la pena recordar, que la metodología cualitativa presenta una cierta tensión entre el mundo conceptual y el empírico, donde la inducción y la deducción no se diferencian nítidamente sino que se entrecruzan.

\section{Participantes}

La encuesta se realizó con una muestra de 46 universitarios de ambos sexos, que representan a la población de interés. El tema de las drogas no se aborda con facilidad en los espacios universitarios. Si bien existe interés generalizado, los jóvenes de ambos sexos aún muestran conductas inhibitorias en relación a abordar el tema de drogas.

Los estudiantes fueron convocados en forma abierta entre los estudiantes de tercer año, que corresponde al Plan Común. Fue una convocatoria abierta pues los estudiantes no están obligados a participar en este tipo de actividades. Se propone la actividad a un grupo grande de estudiantes y ellos determinan si participan o no voluntariamente de la actividad propuesta.

\section{Recolección de datos - instrumentos y procedimientos}

Los datos fueron recolectados a través de grupos de discusión que ocurrieron durante la actividad de 
taller denominada "El fenómeno de las drogas en las universidades de América Latina". La actividad ocurrió durante dos días y contó con la participación de 46 estudiantes.

Los temas orientadores de los grupos de discusión fueron: factores relacionados al uso de drogas; las drogas como problema social; el hombre, un ser dependiente; factores relacionados a la utilización de drogas; valores para vivir; hábitos de vida saludable y el consumo de alcohol en el medio boliviano y en las universidades. El taller como espacio de aprendizaje, fomentó la discusión grupal a partir de exposiciones con ayuda de medios audiovisuales. Las preguntas que surgieron como reflexiones individuales compartidas, permitieron conocer los puntos de vistas que los jóvenes poseen sobre el consumo de alcohol y la importancia que tienen tanto los valores como los hábitos de vida saludable, en la actitud que asumen los jóvenes frente al peligro del alcohol y otras drogas. La discusión grupal facilitó la libre expresión y la comparación entre distintas posiciones de los jóvenes. De esta manera, la discusión dio lugar a que afloraran las distintas interpretaciones y las valoraciones críticas sobre el uso de alcohol. El taller fue conducido por la docente con el apoyo de un grupo de jóvenes que se habían interesado y formado en la temática, desde el inicio de la investigación. La actividad del taller fue filmada y grabada en su integridad, en un CD.

Se utilizó la encuesta diseñada por la investigadora y validada por un panel de expertos, en la perspectiva de establecer un cruce o un "continuo interactivo"(4) en la lógica de una interpretación cualitativa a través de variables de análisis, utilizadas para organizar la información.

El instrumento de recolección de datos fue diseñado como un instrumento sencillo de aplicar $y$ que obtuviera información sobre el problema objeto de estudio. Constaba de tres apartados: contexto del país y universidad; factores de riesgo y protección; y medidas con participación de las y los jóvenes. El primer apartado está compuesto de preguntas semi-abiertas con opción explicativa, respecto a tolerancia social de consumo y adicción; consumo de alcohol y conducta; consumo de alcohol por género en universitarios. El segundo apartado con preguntas semi-abiertas y abiertas en igual proporción, en relacionadas con el factor de mayor influencia en el consumo de alcohol; los valores que se eligen para una vida saludable; los hábitos de vida saludable que les permiten enfrentar el consumo de alcohol; y el tercer apartado con preponderancia de preguntas semi-abiertas, enfocado la relación del consumo de alcohol versus el desempeño académico; pertinencia de un programa de prevención y valores y actitudes requeridos para los jóvenes a cargo del programa.

\section{Análisis de datos}

Se organizaron y clasificaron los conceptos, categorías y respuestas de los jóvenes universitarios. El hilo conductor se centra en los valores y hábitos de vida saludable, a partir de los cuales se teje el entramado que permite retomar los diálogos de los grupos de discusión, las respuestas obtenidas en las encuestas y las valoraciones referenciales de los informantes clave, para ir armando los discursos que sostienen el análisis de contenido.

La docente al ser parte del mundo que estaba estudiando va a describir los sucesos observados de forma reflexiva y narrativa. Tal como fueron vividos y registrados, con las reacciones personales que son parte constitutiva del relato; pero, con la finalidad de alcanzar alguna forma de generalización.

\section{Consideraciones éticas}

La investigación se respalda en la Resolución de Carrera No 177/2008 DE 25 de septiembre de 2008, aprobada por el Consejo de Carrera de la Facultad de Humanidades y Ciencias de la Educación de la Universidad Mayor de San Andrés - Bolivia.

\section{Resultados}

El análisis se sustenta en cuatro categorías que reflejan los valores y hábitos saludables de los estudiantes relacionados al consumo de alcohol.

\section{Los jóvenes y la tolerancia social al consumo de alcohol}

En esta primera categoría los estudiantes relataron que el contexto social no sólo permite sino también motiva e incita al consumo de alcohol.

La sociedad en la que vivimos tiene costumbres y tradiciones que siempre derivan en el consumo de alcohol. (Universitaria de 21 años)

Las afirmaciones registradas permiten aseverar que según la opinión de los participantes la sociedad boliviana es tolerante, liberal y permisiva con el consumo de alcohol por parte de los universitarios.

Porque dentro de la sociedad boliviana cualquier ocasión es motivo de consumo de bebidas alcohólicas, en su mayoría. (Universitaria de 28 años) 
A veces cuando asistimos a una fiesta, un amigo muy lleno de estímulos propios del alcohol nos exige beber una copa, aduciendo que lo hagamos porque lo queremos. (Universitaria de 21 años)

El inicio en el consumo de alcohol en el caso de los universitarios, es un "ejercicio de descubrimiento" e interacción social con sus pares; que se va transformando en exigencias de mayor consumo en menor tiempo, pero que no está vinculado a alcanzar niveles de dependencia física del alcohol.

Porque es bien visto que se beba alcohol desde temprana edad, en especial en los hombres (Universitaria de 23 años).

El consumo de alcohol empieza como un juego para ser aceptado por sus amigos, lo que termina mal. (Universitario de 20 años).

\section{Razones para el consumo de alcohol en los universitarios}

En esta categoría, los estudiantes expresaron su comprensión sobre las razones psicológicas, sociales y de género que llevan al consumo de alcohol.

Los valores generan un marco de actuación regulador frente al consumo de alcohol, para un grupo mayoritario de jóvenes. Sin embargo, existen universitarios con expectativas positivas frente al consumo. No sólo significa que no alcanzan a dilucidar los peligros del consumo de alcohol, sino que en algunos casos, se percibe la ausencia de un diálogo interno del universitario, como parte del proceso reflexivo individual.

Existen infinidad de razones, para el consumo de alcohol en universitarios, las principales serían problemas familiares, emocionales, sentimentales, depresivos y generalmente, porque los jóvenes buscan un consuelo en el alcohol, al sacar lo que están guardando por dentro, rabia, furia, rencor y odio a sus situaciones vividas. (Universitario de 23 años).

La juventud es un periodo de experimentación interesante. Beber para algunos constituye un reto, en la lógica de 'a ver que no te atreves; si no lo haces, eres cobarde'. (Universitario de 22 años).

Las razones del consumo de alcohol son semejantes en hombres y mujeres. Tanto los universitarios como las universitarias ingieren alcohol por problemas familiares, emocionales, sentimentales, depresión, aburrimiento, falta de poder e inconformidad con el medio y con ellos mismos.

Los hombres jóvenes consumen más porque tienen problemas sentimentales o problemas familiares, La tolerancia sobre este tema del alcoholismo no es buena consejera, por esta razón debemos hacer algo más a lo que ya hemos hecho, pues más adelante no tendrá solución. (Universitaria de 20 años)
Los jóvenes consumen alcohol porque no tienen mucho control y además son los que se quedan hasta más tarde. (Grupo de universitarias entre 21-24 años)

El consumo de alcohol modifica sustancialmente, las formas de comportamiento habituales de los universitarios(as). Existe una percepción unánime en los universitarios varones, que el consumo social de alcohol no guarda conexión con la adicción al alcohol. Los valores como un ejercicio de la libertad son visualizados como contenedores que generan auto-dominio en el comportamiento social.

Los jóvenes consumen alcohol por pasar el tiempo, olvidarse del mundo o lo hacen para no estar aburridos. (Grupo mixto de universitarios entre 20-25 años).

\section{Los valores y hábitos - factores de protección en universitarios}

Los estudiantes también hicieron descripciones detalladas que indican como los valores y hábitos de vida saludable se constituyen en factores de protección ante el consumo de alcohol. De igual manera, las amistades son un factor de riesgo continuo para los jóvenes.

El amor propio es el primer valor, porque si uno se respeta a sí misma, ve a su cuerpo y a su salud como algo importante y digno. Otro valor es el amor hacia los demás porque cuando se bebe alcohol se daña a las personas que nos rodean. (Universitaria de 23 años).

Los valores afianzados en la personalidad se constituyen en los factores de protección fundamentales. Los principales valores que les permiten enfrentar adecuadamente el consumo de alcohol en orden de prioridad, son los siguientes: el amor y respeto por sí mismo, la tolerancia, la autoestima, la responsabilidad. La solidaridad, la bondad y la alegría son vistas como valores menos representativos. Un énfasis especial le conceden a la capacidad reflexiva de cada joven $\mathrm{y} / \mathrm{o}$ jovencita.

Los valores bien afianzados, les permite rechazar las influencias de los demás. (Universitario de 20 años).

La tolerancia es necesaria para que los jóvenes líderes de un grupo determinado respeten a otro que tal vez no quiere ir con ellos a consumir alcohol. (Universitario de 22 años).

Los hábitos de vida saludable juegan un papel preeminente en sus vidas. Los más reconocidos son el deporte, el estudio, la música, el teatro y la lectura, visualizados como generadores de la capacidad de pensar por sí mismos, dilucidar y elegir - fruto del discernimiento - en las y los estudiantes.

Cuando una persona se encuentra ocupada en una actividad divertida: hacer deportes u otra recreación, se le reducen 
las ansías y la curiosidad de consumir bebidas alcohólicas. (Universitaria de 22 años)

Los hábitos de vida saludable son un candado para resistir la influencia del entorno a consumir alcohol. (Universitario de 23 años)

Una personalidad ya definida y un estilo de vida saludable son los factores que ayudan a resistir la presión de otros con relación al consumo de alcohol. Existen muchos jóvenes que valoran mucho su vida, por eso buscan alternativas para poder desarrollar su personalidad, como por ejemplo, el deporte, la música, el teatro, la lectura. (Universitaria de 21 años)

Una puede tener los hábitos de vida saludable muy enraizados o consolidados, pero puede en algún momento ser débil ante el consumo de alcohol ya sea por una depresión extrema o por insistencia de sus amigos. (Universitaria de 21 años)

El deporte es considerado por los universitarios varones y mujeres como un hábito emblemático de vida saludable; vinculado al consumo posterior de bebidas alcohólicas, desvirtúa su sentido modélico. Los hábitos de vida saludable con los que se identifica la población universitaria son el deporte en primer término, seguido del estudio y por último, las actividades recreativas.

En nuestro medio se escucha frecuentemente el eslogan 'si al deporte, no a las drogas', pero en algunos casos se presentan situaciones donde después de jugar futbol se comienza a ingerir alcohol. Simplemente por ganar el partido o por perderlo, en estos casos es relativo. (Universitario de 23 años)

En relación a la asociación entre consumo de alcohol y desempeño académico, no hubo un pronunciamiento claro de los universitarios, aunque las autoridades universitarias si establecieron un vínculo causal de consumo de drogas y desinterés por el estudio.

\section{Razones para crear un Programa Universitario de Prevención del Uso Indebido de Drogas}

Los universitarios evidenciaron argumentos que sostienen la creación de un programa de intervención direccionado a esta población.

Frente al abuso de alcohol o su posibilidad se requiere el compromiso social de la universidad. (Universitario de 22 años)

El empoderamiento de los universitarios constituye un mecanismo de protección a la ingesta de alcohol. El colectivo asume que el empoderamiento que ha alcanzado -gracias a los procesos formativos de nivel reflexivo en el que están insertos- le ha permitido interrogarse sobre sus pensamientos y acciones. El empoderamiento los vuelven más seguros de ellos mismos, más firmes en sus juicios, más dispuestos a la responsabilidad y control de sus vidas y a la toma de decisiones propias.
Como universitarios tenemos que ayudar a la sociedad, ayudar a otros jóvenes a darse cuenta que el alcohol y las drogas son situaciones que nos desmejoran en nuestra salud, respeto y confianza. (Universitaria de 22 años)

Los jóvenes al ser partícipes de programas de prevención e involucrarse en la temática, sean o no consumidores, reflexionan y pueden tomar mejores decisiones acerca de sus vidas y ayudar a sus compañeros. (Universitaria de 25 años)

El equipo de jóvenes gestores del Proyecto de Prevención debe ser perseverante para no desistir en la lucha contra las drogas ya que no es algo sencillo de hacer. También deber ser moderados para dar un ejemplo con su vida. Deben ser respetuosos para tratar con la debida dignidad a aquellos que se encuentran enfrascados en el mundo de las drogas. (Universitaria de 23 años)

Las autoridades universitarias consideraron fundamental el modelado del docente - con un conjunto de valores y conductas - para contribuir al ejercicio de la responsabilidad en los jóvenes.

\section{Discusión}

El Estudio reafirma que la universidad es un espacio propicio para desplegar y acrecentar valores y hábitos de vida saludable. Las universidades permiten que los jóvenes cultiven las capacidades argumentativas $y$ reflexivas para tomar decisiones afirmativas en relación al alcohol.

Se ha encontrado en la literatura actual, que existe un conjunto de valores, actitudes y maneras de actuar que no se aprende si nadie se toma la molestia de enseñarlo(5).

Los pilares para ser, hacer, actuar y convivir(6), en un joven, se construyen en el primer núcleo de referencia que es la familia. Algunos autores sostienen que la familia juega un rol central en el logro de la salud psíquica, el equilibrio emocional, la madurez, la inteligencia emocional y la capacidad de aprendizaje de los jóvenes ${ }^{(7)}$.

Estudios similares realizados con jóvenes colombianos $^{(8)}$ demostraron que el consumo de alcohol en jóvenes universitarios es superior a la de mujeres universitarias. Las condiciones socio-culturales y el sesgo de género, determinan un consumo moderado o abstención de consumo de alcohol en las mujeres. En el caso del presente estudio, el empoderamiento femenino en auge, es la causa fundamental de la distancia cuidada, de las universitarias en relación al consumo de alcohol.

Los móviles para la ingesta de alcohol en los jóvenes universitarios están ligados a la necesidad de reforzar la autoestima depreciada y la interacción y aceptación 
social de sus pares. En la misma dirección se encuentran los resultados obtenidos con jóvenes universitarios de Cali, Colombia(9).

El Estudio coincide con la investigación efectuada con los estudiantes de enfermería de la Universidad Mayor de San Andrés ${ }^{(10)}$, sobre las actitudes tolerantes y permisivas de la sociedad boliviana con relación al uso de alcohol y la necesidad de generar programas de prevención abiertos e integrados en la comunidad universitaria.

Un importante reto para la universidad boliviana y para las universidades de América Latina, es promover la continuidad en la realización de investigaciones cualitativas que refuercen la temática que estimulen a las y los universitarios a ejercitar el pensamiento crítico con sus iguales y compañeros, los alienten en la posibilidad del diálogo con otros/as; les permita reconstruir su forma de verse a sí mismos, de tal modo que actúen en el mundo posesionados de un conjunto de valores para vivir con dignidad y autoestima.

Un Programa de Prevención del Uso Indebido de Drogas abierto a todas las Facultades, gozaría de plena aceptación, entendido como un proceso educativo vinculado a la formación y/o afianzamiento del carácter en los jóvenes. Lo conciben como parte de la tarea de la universidad de comprometerse con una educación que proporcione a los individuos los elementos para alcanzar una mayor independencia de juicio, de deliberación y de diálogo con capacidad de escucha. El Programa que tendría la finalidad de coadyuvar a la toma de decisiones informadas en universitarios/as, requiere de la participación decisiva de jóvenes líderes, portadores de valores dignos de ser imitados.

\section{Conclusiones}

El retorno de la esperanza a la educación en general y a la educación universitaria, será posible si la educación nuevamente se hace responsable de formar las actitudes de las personas, en el mejor sentido, si de verdad compromete su accionar en inculcar valores inmateriales en un mundo donde el poder de los bienes materiales, ejerce una influencia decisiva y hasta cierta fascinación en los individuos. Necesitamos que la educación se haga cargo de los valores que queremos no sean desplazados o desaparezcan sino que sean preservados en el futuro. Hace falta que la educación retome el significado primigenio de lo que significa educar: extraer de la persona lo mejor que lleva dentro.

Si la investigación ha girado alrededor de valores y hábitos de vida saludable, ésta nos ha relanzado más allá: a la relación del consumo de alcohol con la salud pública; a la necesidad de rediseñar los espacios universitarios como entornos saludables; a convocar a las y los docentes como fuentes de identidad; a revisar los fundamentos morales y el rol modélico de nuestra práctica para dinamizar nuevas actitudes y prácticas en las y los universitarios; y a comprender que la salud y la educación se entrelazan en la temática de prevención del consumo de alcohol, construyendo una red compacta de relaciones de dependencia y mutuas determinaciones. Requerimos en las universidades que las Carreras ligadas a la Educación y la Salud establezcan mecanismos de relacionamiento eficaces, para generar programas preventivos e integrales de mayor alcance.

El Estudio también revela cómo los mandatos de género, no siempre explícitos, se manifiestan en diversos niveles en el universo simbólico de la vida cotidiana de los jóvenes. Este hecho dificulta la actitud crítica de éstos en relación a las interacciones sociales con el alcohol. Abre una perspectiva distinta en relación al empoderamiento de las jóvenes universitarias que revela cuestionamientos directos sobre la vida personal, la dimensión externa de la vida social y una autoestima entretejida de valores racional y libremente asumidos, que les permiten tomar decisiones propias con relación a los diferentes argumentos, que se les presentan para consumir alcohol.

En el mundo actual, existe una responsabilidad pública de la universidad de definir los valores que están en el centro de su actuación y proporcionar a los jóvenes las pautas para que ellos, puedan optar en relación a éstos. En este sentido, la universidad requiere comprometer su discurso y su acción en la dirección de promover valores y hábitos de vida saludable, a través de la incorporación de Programas de Prevención que se constituyan en orientadores e inspiradores de los jóvenes.

\section{Agradecimientos}

Agradecemos a la Comisión Interamericana para el Control del Abuso de Drogas/CICAD de la Secretaria de Seguridad Multidimensional/SSM de la Organización de Ios Estados Americanos/OEA, la Secretaria Nacional de Políticas sobre Drogas/SENAD do Gabinete de Seguridad Institucional/Brasil, la Escuela de Enfermería de Ribeirao Preto de la Universidad de Sao Paulo y Centro Colaborador de la Organización Mundial de la Salud para el Desarrollo de la Investigación en Enfermería, la población representada en los estudios de investigación, 
bien como a las autoridades de las universidades representadas por los participantes del Programa En-Line de Especialización en Investigación sobre el Fenómeno de las Drogas, periodos 2006, 2007, 2008 y 2009.

\section{Referencias}

1. Alcaraz Del CF. Informe Final. Estudio comparativo urbano. Consumo de alcohol, tabaco, cocaína y otras drogas en Bolivia 1992-1996-1998-2000-2005. La Paz: CELIN Centro Latino Americano de Investigación Científica; 2005.

2. Centro Latino Americano de Investigación Científica (CELIN). Uso indebido de drogas en estudiantes universitarios en Bolivia. La Paz: Centro Latino Americano de Investigación Científica; 2008.

3. Gadamer HG. Acotaciones hermenéuticas. Madrid: Trotta; 2002. 299 p.

4. Arnold DY. Metodología en las ciencias sociales en la Bolivia postcolonial: Reflexiones sobre el análisis de los datos en su contexto. In: Yapu M, coord. Pautas metodológicas para investigaciones cualitativas $y$ cuantitativas en ciencias sociales y humanas. La Paz: Serie PIEB metodológica; 2006. p. 7-101.

5. Camps V. Creer en la educación: La asignatura pendiente. Barcelona: Ediciones Península; 2008.

6. Delors J. La educación encierra un tesoro. Madrid: Ediciones Unesco; 1996.

7. Kliksberg B. El contexto de la juventud en América Latina y el Caribe: los grandes interrogantes. In: Fundación W.K. Kellogg - Fundación Petrópolis (Eds.). Asociándose a la Juventud para Construir el Futuro. Sao Paulo: Editorial Petrópolis; 2006. p. 32-9.

8. Londoño C, García W, Valencia S, Vinaccia S. Expectativas frente al consumo de alcohol en jóvenes universitarios colombianos. In: Anales de Psicologia diciembre 2005 [acesso 08 jan 2009]; 21(2):259-67. Disponível em: http://revistas.um.es/analesps/issue/ view/2321

9. Cáceres D, Salazar I, Varela M, Tovar J. Consumo de drogas en jóvenes universitarios y su relación de riesgo y protección con los factores psicosociales. Bogotá: Universidad Psychol; 2006. p. 521-34.

10. Flores IEE, Luis MAV. Uso y actitudes relacionados a las drogas en las estudiantes de enfermería de la Universidad Mayor de San Andrés. Rev. Latino-Am. 